\title{
Preliminary Analysis in MINERvA's Nuclear Targets for CCQE-like Events
}

\author{
2018-06-18
}




\section{Motivation}

- Neutrino oscillation measurements need to know the neutrino energy to high precision

- Can't observe the neutrino directly

- Reconstruct energy from outgoing particles

- Different interaction types

$\rightarrow$ different reconstructed $\mathrm{E}_{\mathrm{V}}$

- Need precise measurement of

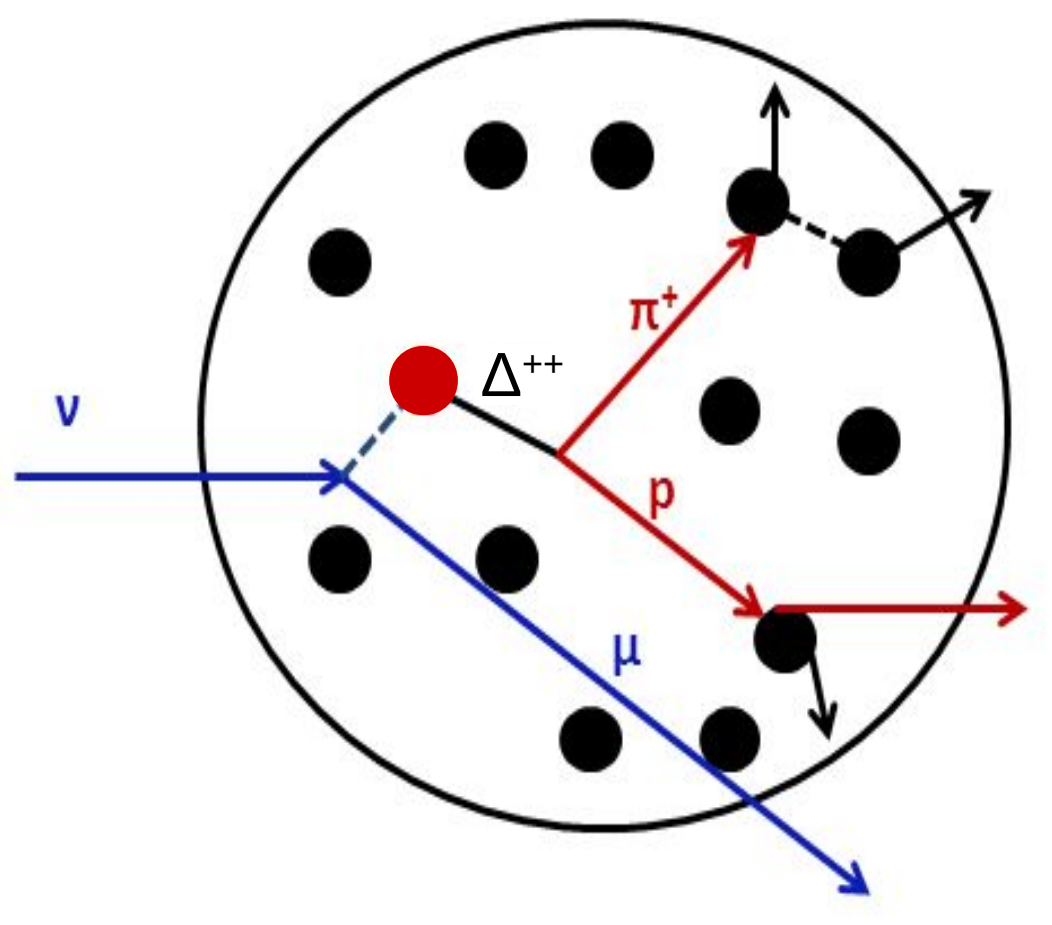
neutrino-nucleus cross section

- A dependence 


\section{MINERvA Detector}
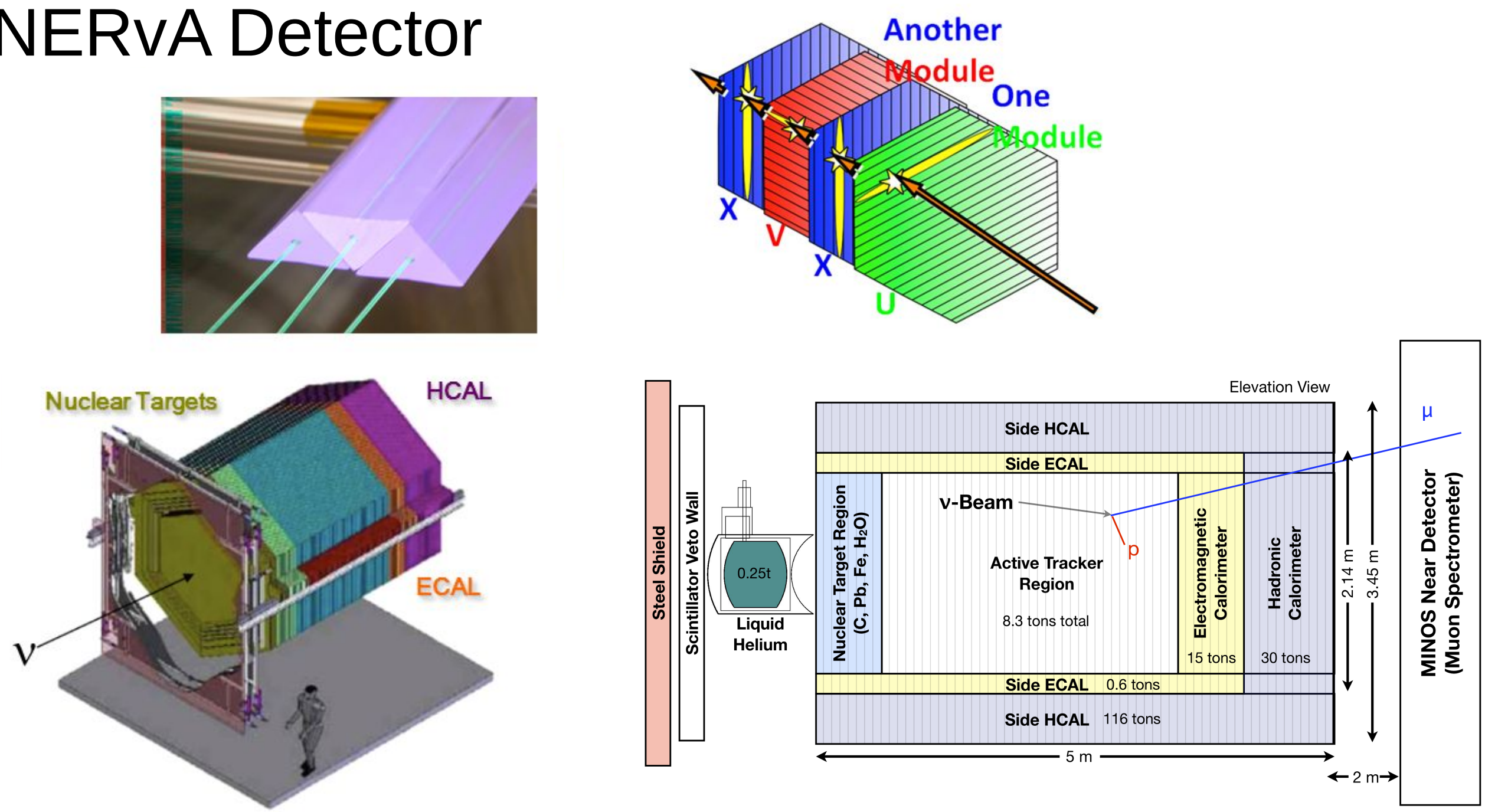


\section{Nuclear Targets}

Neutrino beam direction






\section{Reconstructed Interaction Vertex Position In Nuclear Target Region}

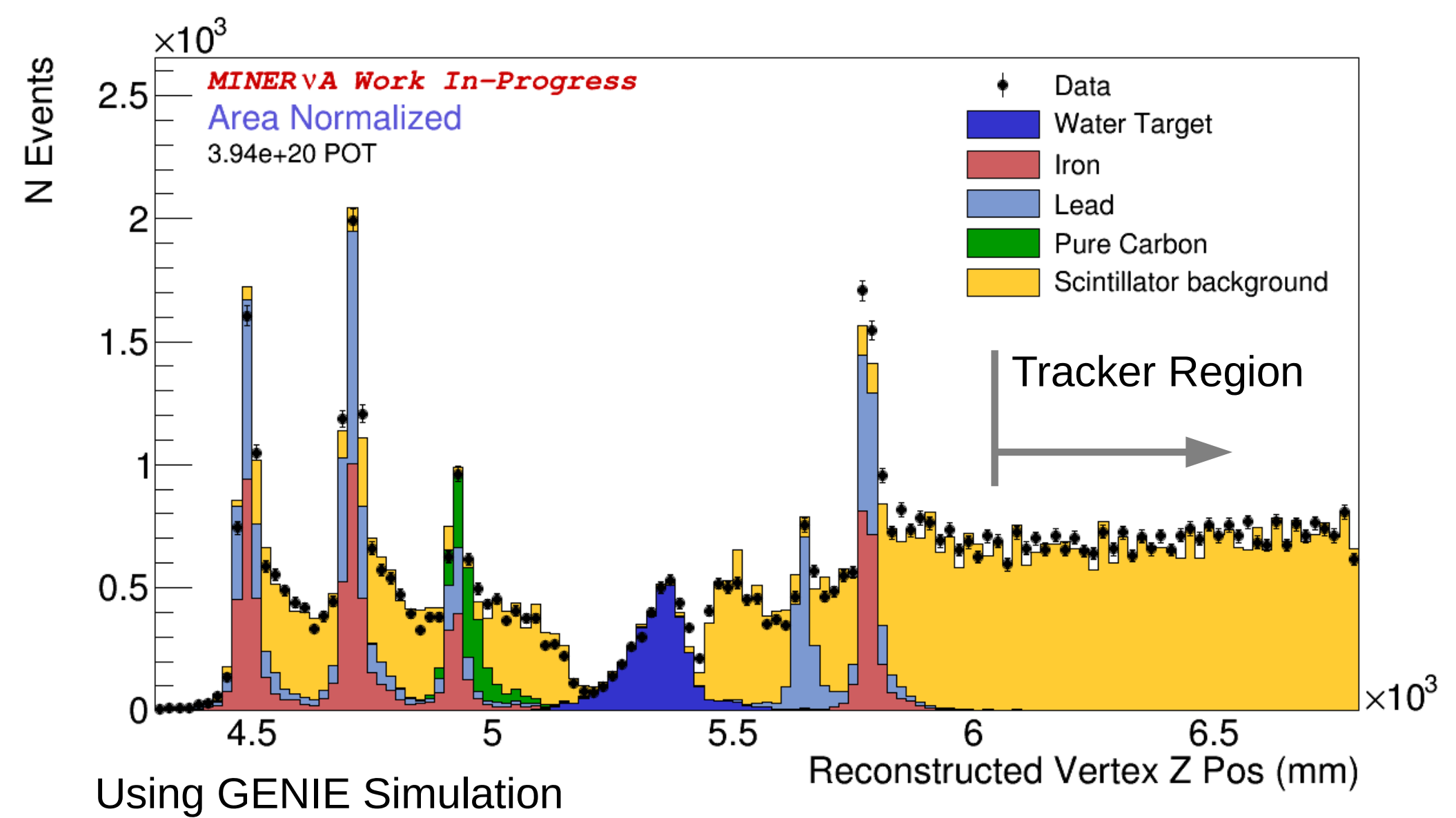




\section{CCQE-like (CCO $\pi)$}

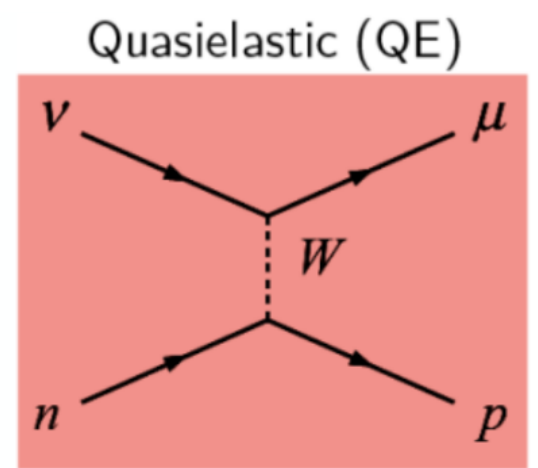

\section{Resonance}

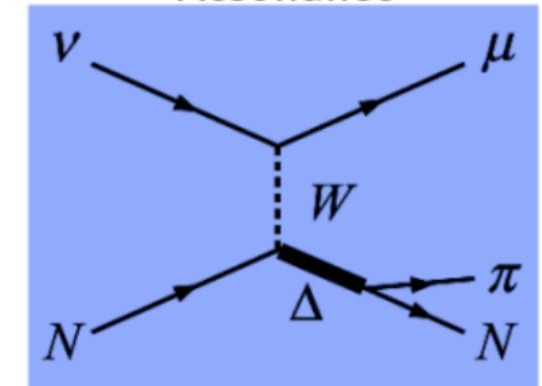

DIS

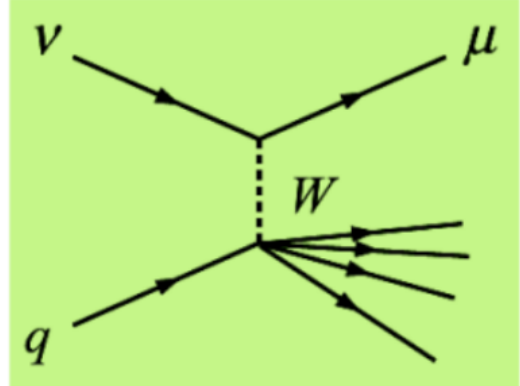

- Pion can be absorbed by nucleus

- Events with pions can look like QE

- Final state:

1 Muon

no mesons

no gammas > $10 \mathrm{MeV}$ (usually come from Pi0)

- More closely matches capabilities of proton-blind detectors 


\section{Muon Momentum}

- Muon is largely unaffected by the nucleus

- $\mathrm{P}_{\mathrm{z}}=$ momentum along neutrino beam direction ( $\mathrm{z}$ direction)

- $\mathrm{P}_{\mathrm{t}}=$ momentum along transverse direction (perpendicular to $\mathrm{z}$ )

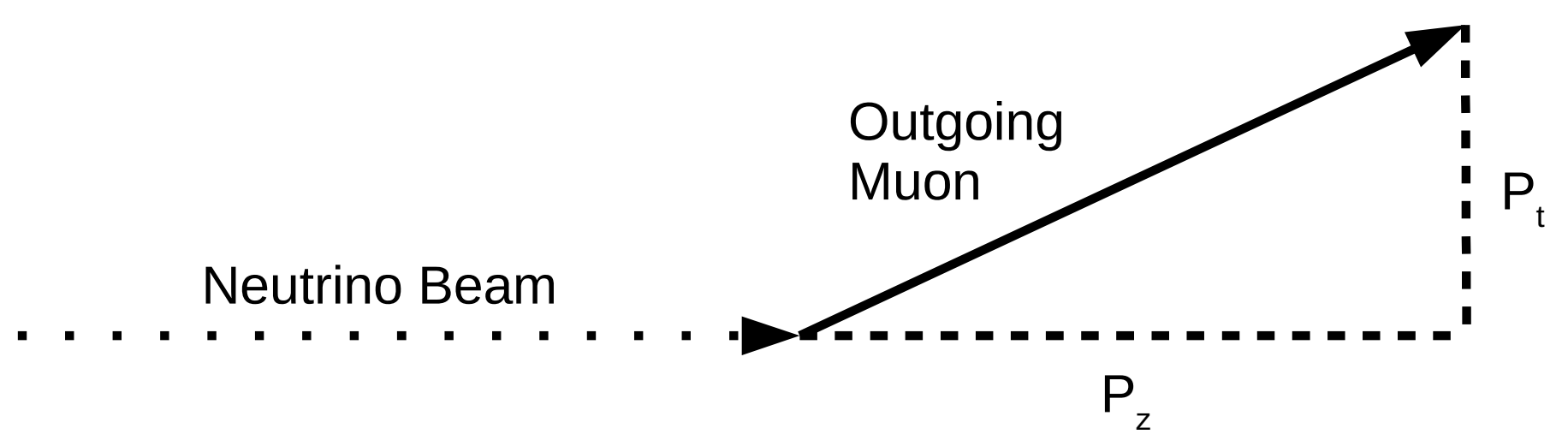




\section{Lead Muon Momentum in Bins of $P_{z}$}
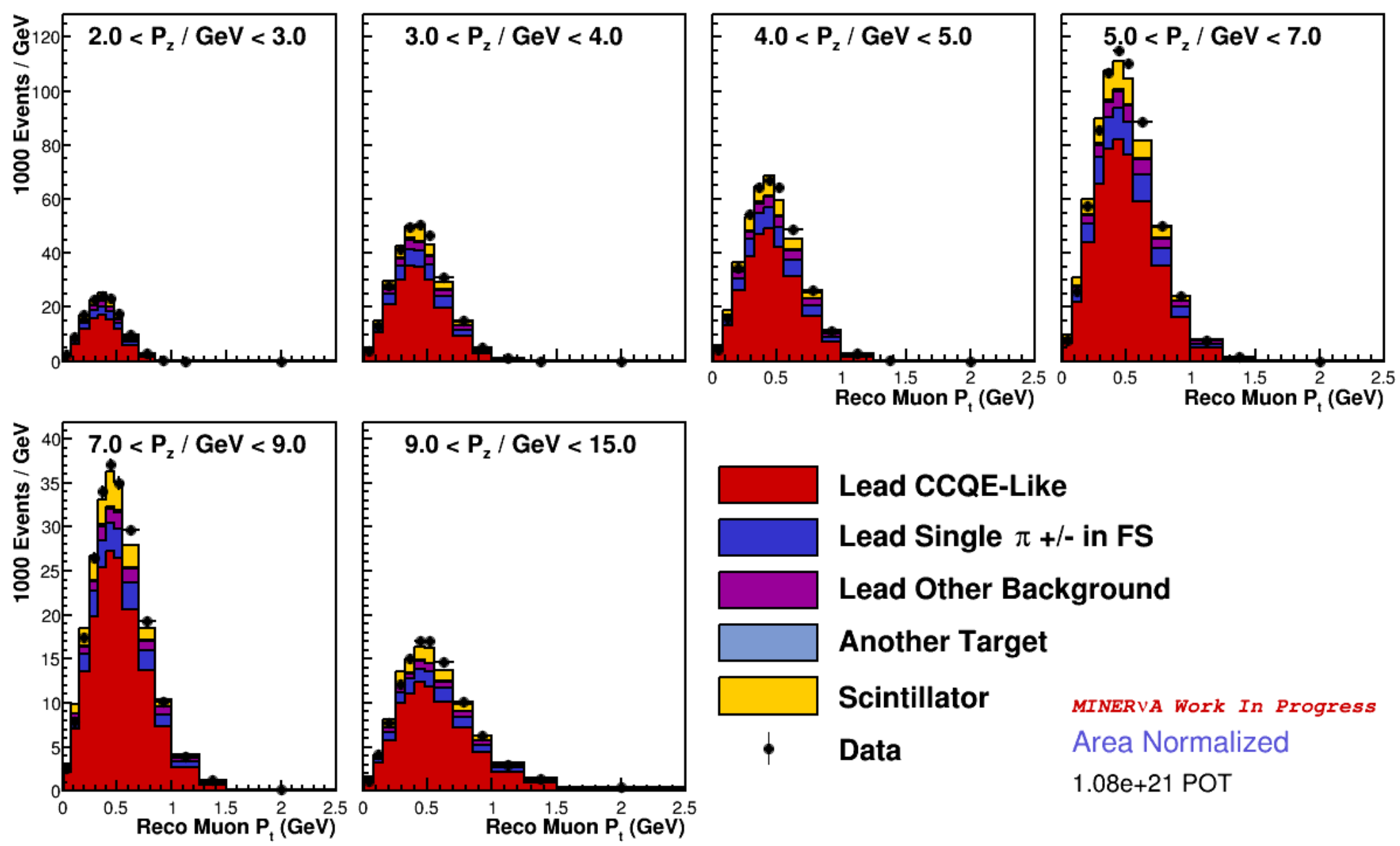

Lead CCQE-Like

Lead Single $\pi+/$ - in FS

Lead Other Background

Another Target

Scintillator

MINERVA Work In Progress

Data

Area Normalized

$1.08 \mathrm{e}+21 \mathrm{POT}$ 


\section{Proton Kinetic Energy}

- Proton Kinetic Energy (KE) found via dE/dX

- Only a portion of events have a reconstructed proton

- Proton needs to create long enough track for reconstruction

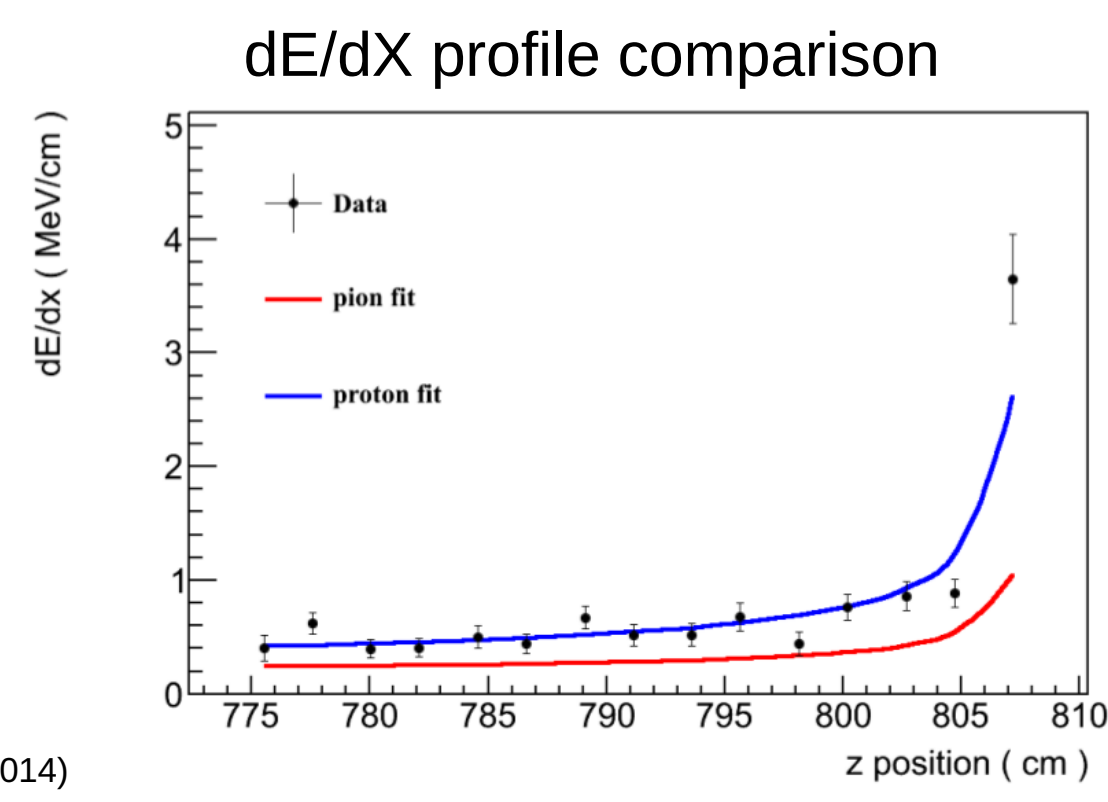




\section{Proton Kinetic Energy}

- Proton Kinetic Energy (KE) found via dE/dX

- Only a portion of events have a reconstructed proton

- Proton needs to create long enough track for reconstruction

- Pions are rejected with two methods:
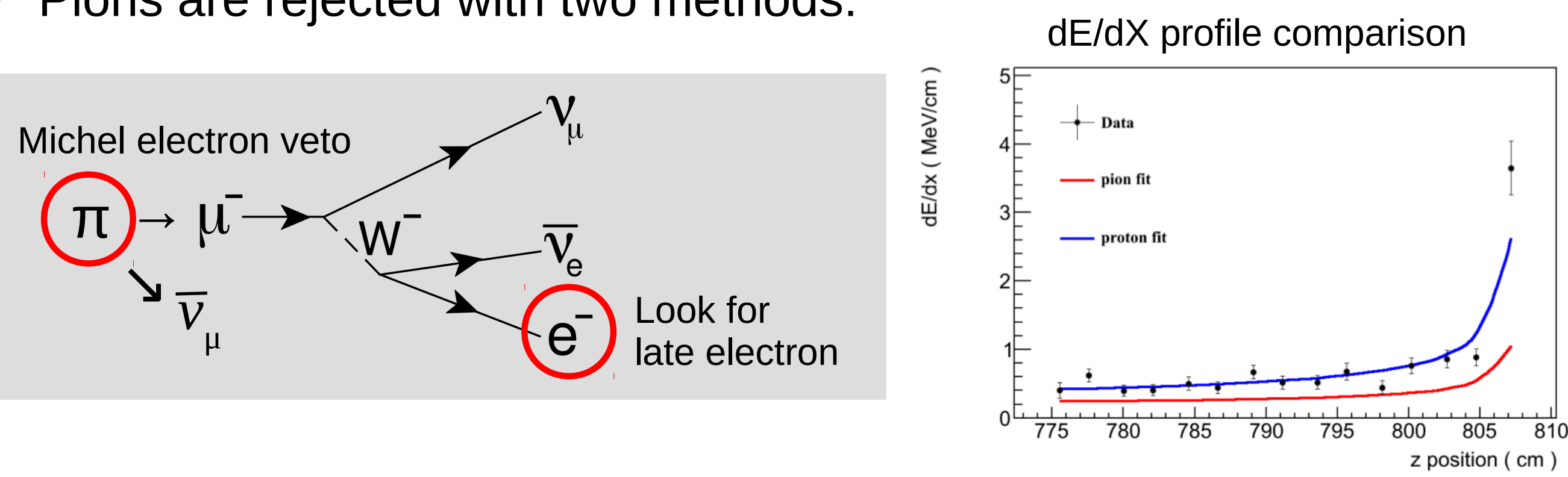


\section{Reconstructed Proton Kinetic Energy in Lead}

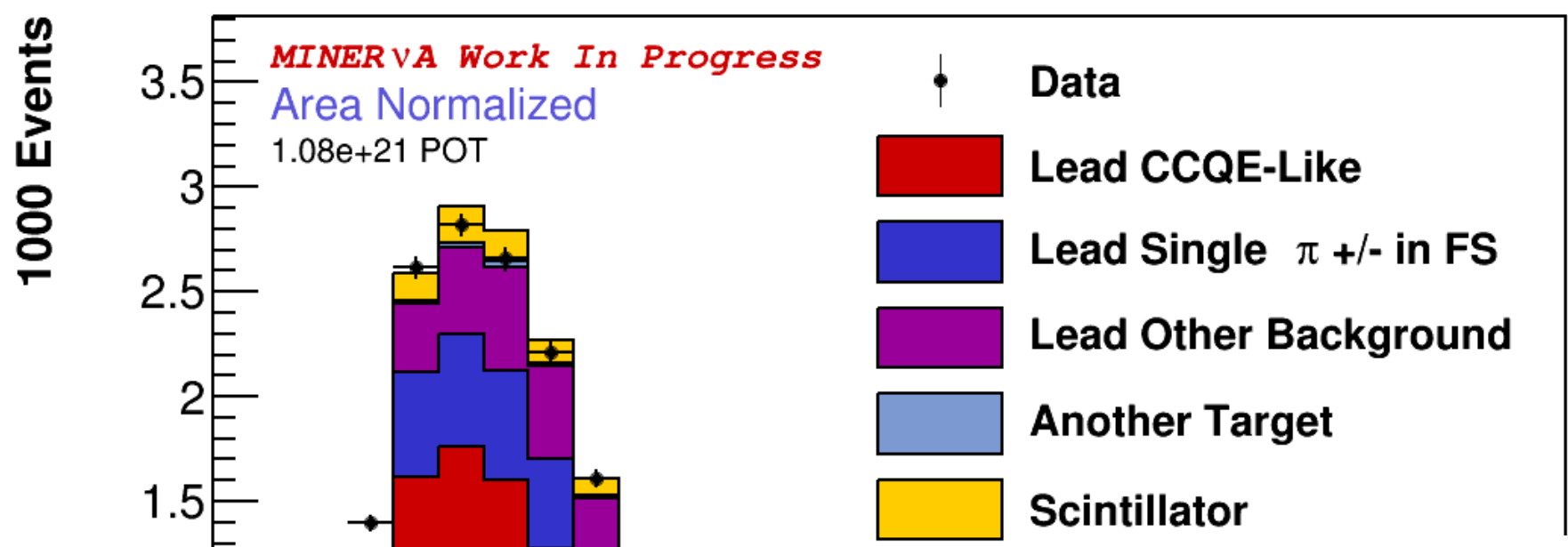

In case of two or more reconstructed protons, pick the highest energy 


\section{Inside the nucleus}

- Fermi motion

- Natural motion of protons and neutrons before interaction

- Energy 100s of MeVs

- Final State Interactions (FSI) can

- Change proton momentum

- Change particle charge

- Absorb pions

- Muon largely unaffected

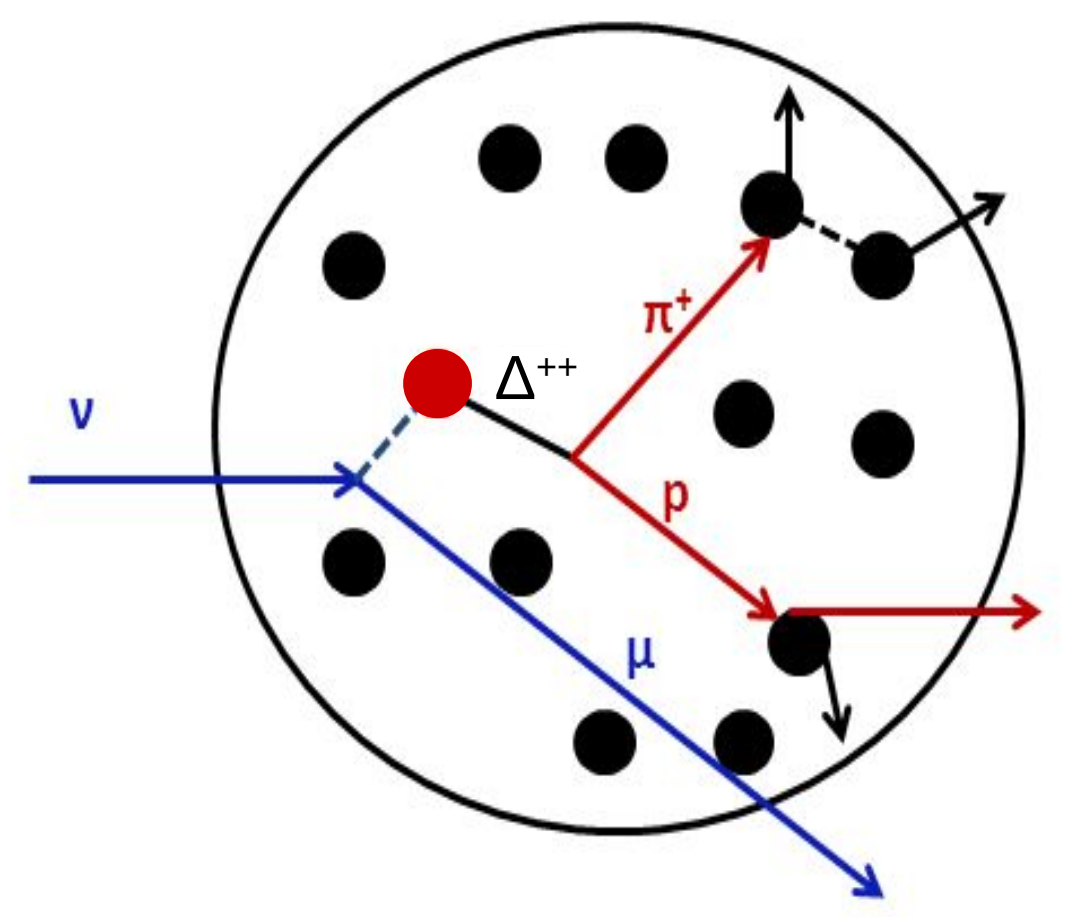




\section{Transverse Momentum Imbalance}

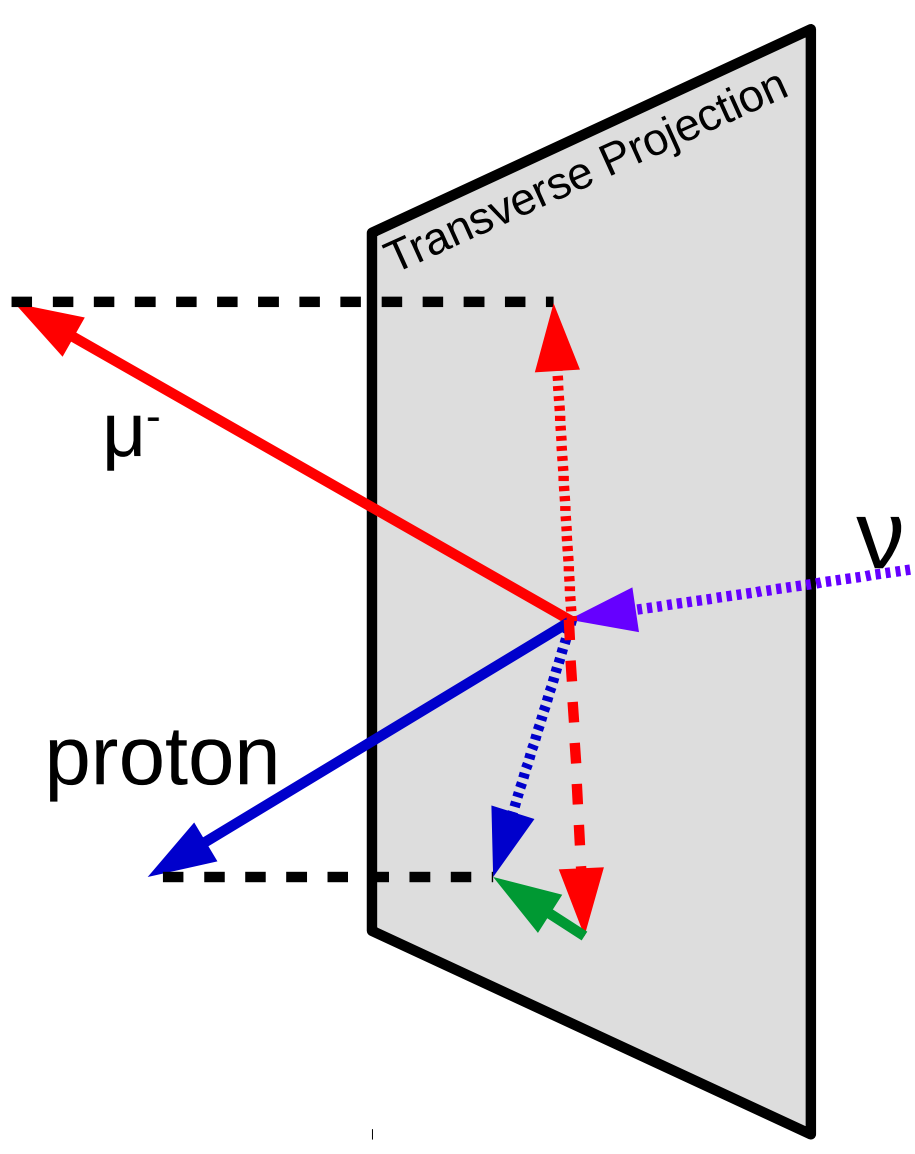

Transverse Projection

Coplanarity

Angle

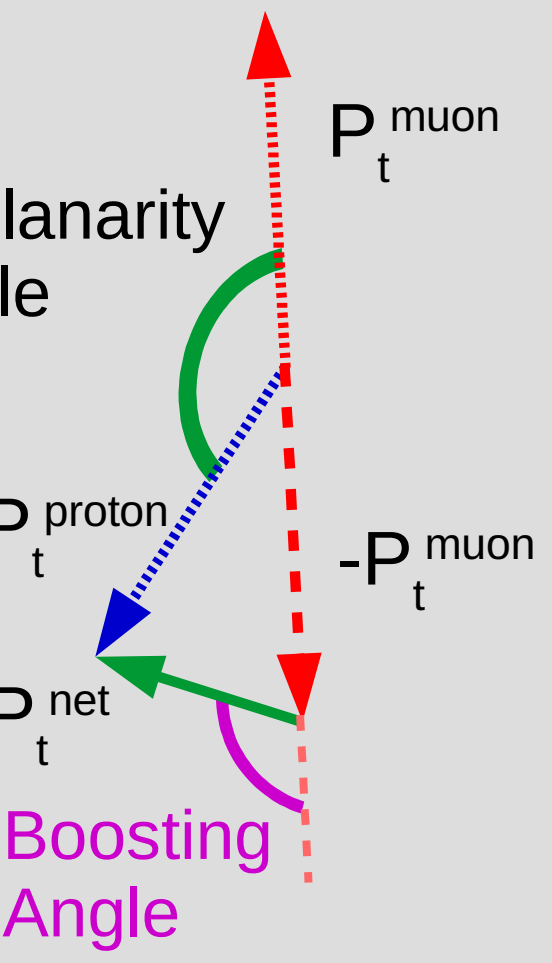

- With no interactions, proton transverse momentum would be opposite muon

- Muon-Proton momentum differences give insight into nucleus

- Coplanarity angle $\rightarrow$ proton deflection

- Boosting angle $\rightarrow$ other FSI effects

[2] A. P. Furmanski and J. T. Sobczyk, Phys. Rev. C 95, 065501 (2017) [3] X. Lu, et al. [MINERvA Collaboration], arXiv:1805.05486 [hep-ex] 


\section{Coplanarity Angle in Lead}

Measures Proton Deflection

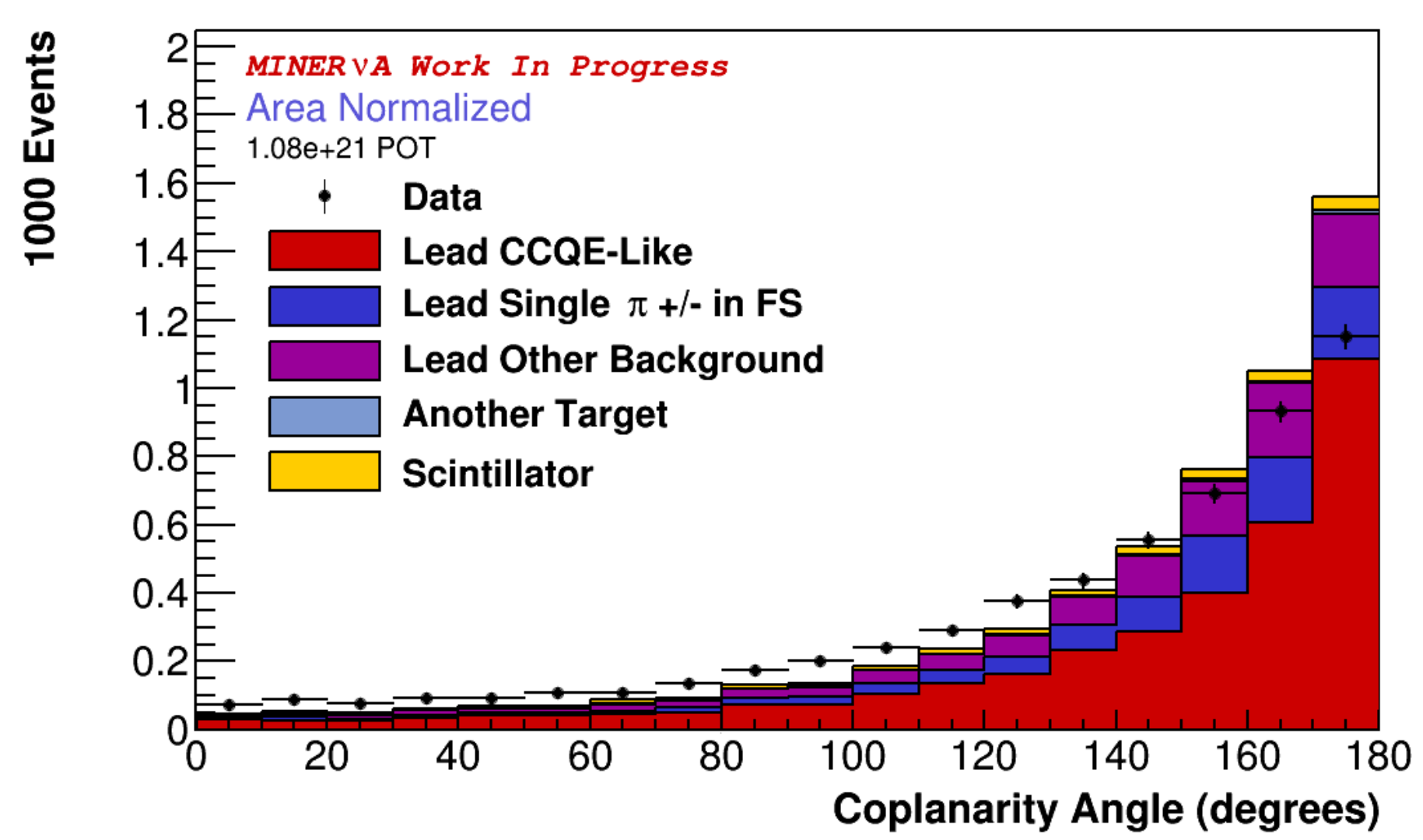

Transverse Projection

Coplanarity Angle

P proton

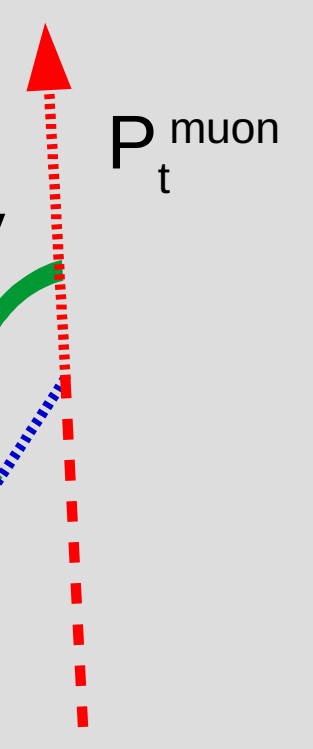




\section{Transverse Boosting Angle in Lead}
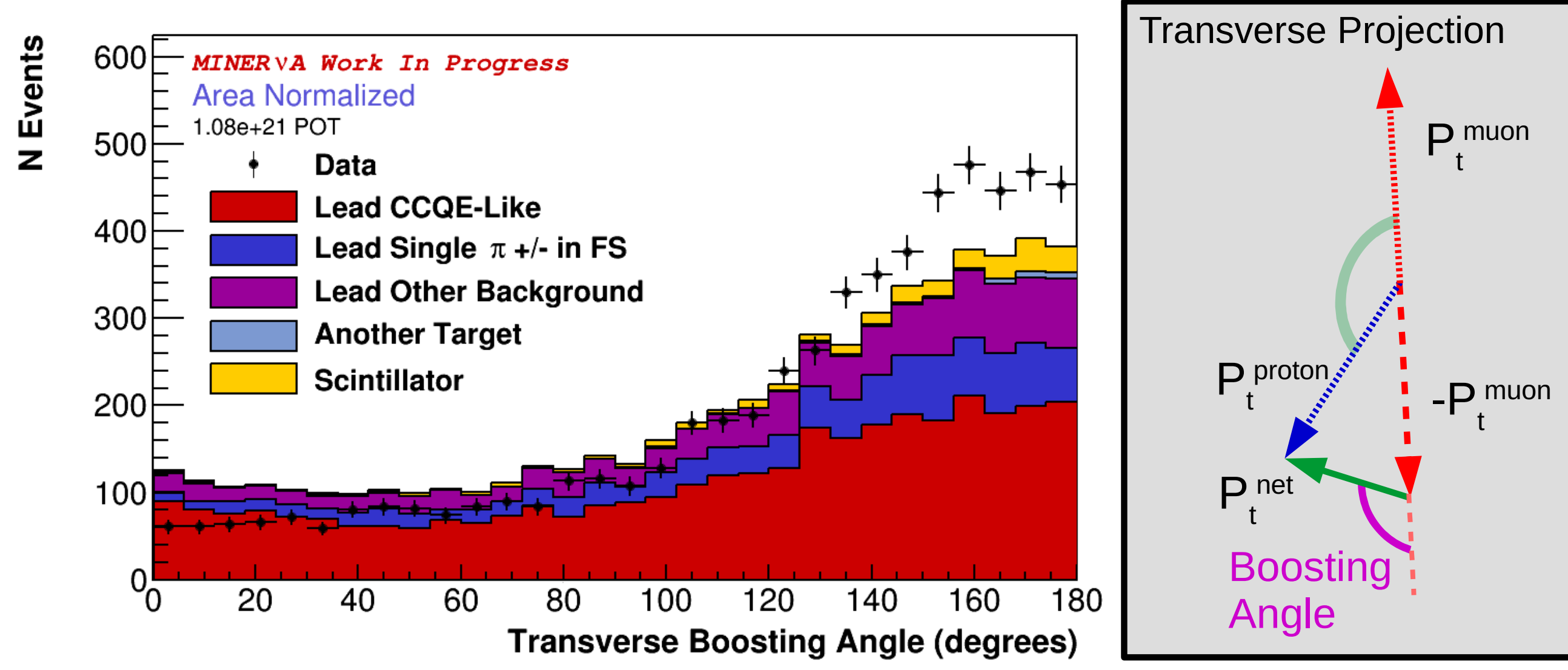


\section{Conclusion}

- NuMI beam's medium energy run provides high statistics

- Mature analysis technique to isolate events in nuclear targets

- Statistically significant differences between data and simulation

- See trend as function of nucleus size

- Double differential cross section

- Results coming soon 


\section{Thank You}

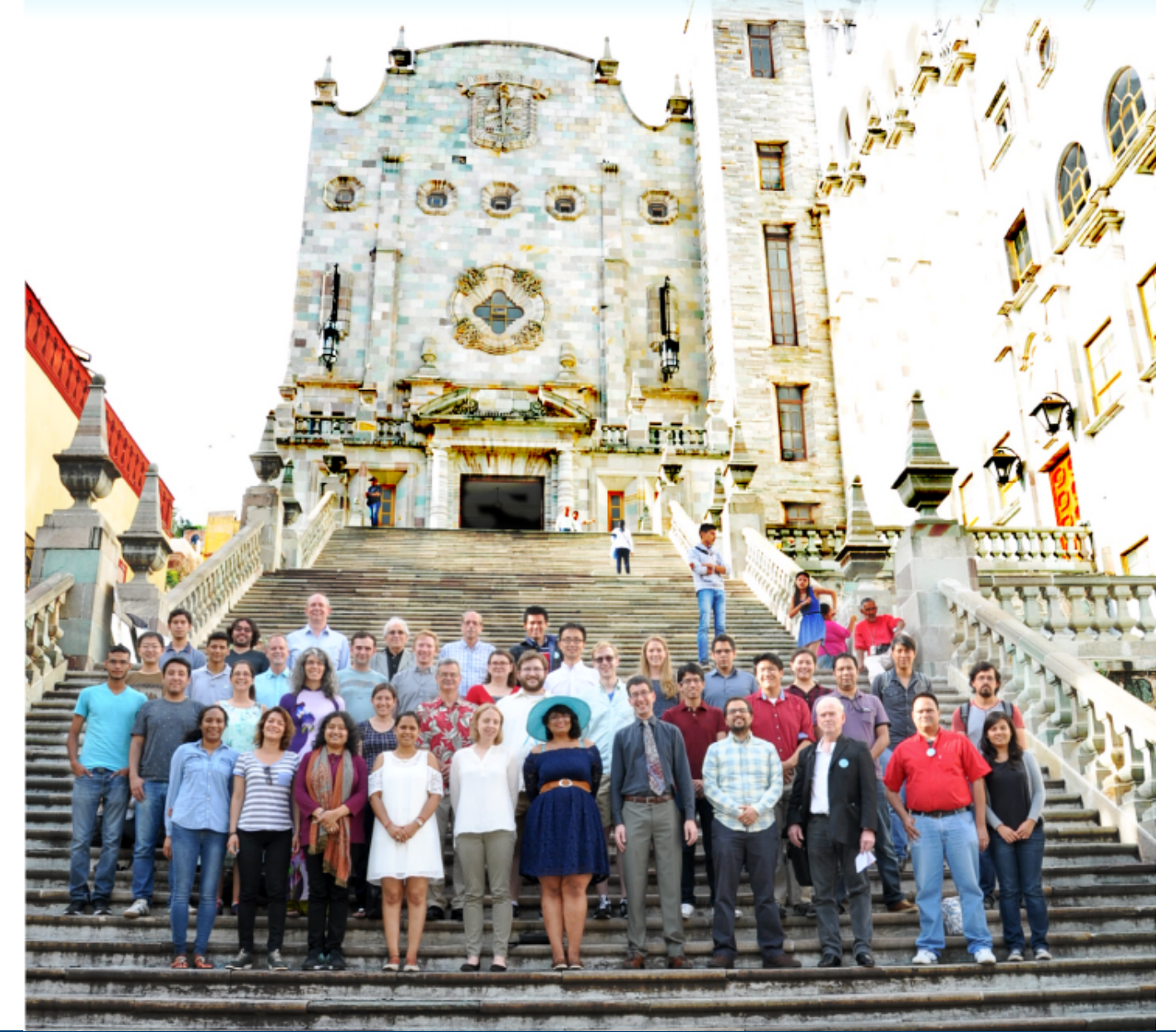




\section{Backup}




\section{Simulation Info}

- Using MnvGENIE-v1 simulation with various changes including

- Reduced pion production

- Random Phase Approximation (RPA) correction

- Valencia 2p2h with tune using MINERvA inclusive scattering data

- No pion prediction

- See [1-6]

[1] C. Andreopoulos et al., Nucl. Instrum. Meth. A 614, 87 (2010), Program version 2.8.4, with private modifications, used here.

[2] A. Higuera et al. (MINERvA Collaboration), Phys. Rev. Lett. 113, 261802 (2014), arXiv:1409.3835 [hep-ex] .

C. Wilkinson et al., Phys. Rev. D 90, 112017 (2014), arXiv:1411.4482 [hep-ex] .

C. Wilkinson et al., In preparation 90 (2015), 10.1103/PhysRevD.90.112017, arXiv:15xx.xxxxx [hep-ex] .

[3] J. Nieves, J. E. Amaro, and M. Valverde, Phys. Rev. C 70, 055503 (2004), arXiv:nucl-th/0408005 [nucl-th].

[4] J. Nieves, I. Ruiz Simo, and M. Vicente Vacas, Phys. Rev. C 83, 045501 (2011), arXiv:1102.2777 [hepph] .

[5] R. Gran, J. Nieves, F. Sanchez, and M. Vicente Vacas, Phys. Rev. D 88, 113007 (2013), arXiv:1307.8105 [hep-ph] .

[6] P. A. Rodrigues et al. [MINERvA Collabtoration], Phys Rev. Lett. 116, 071802 (2016) 


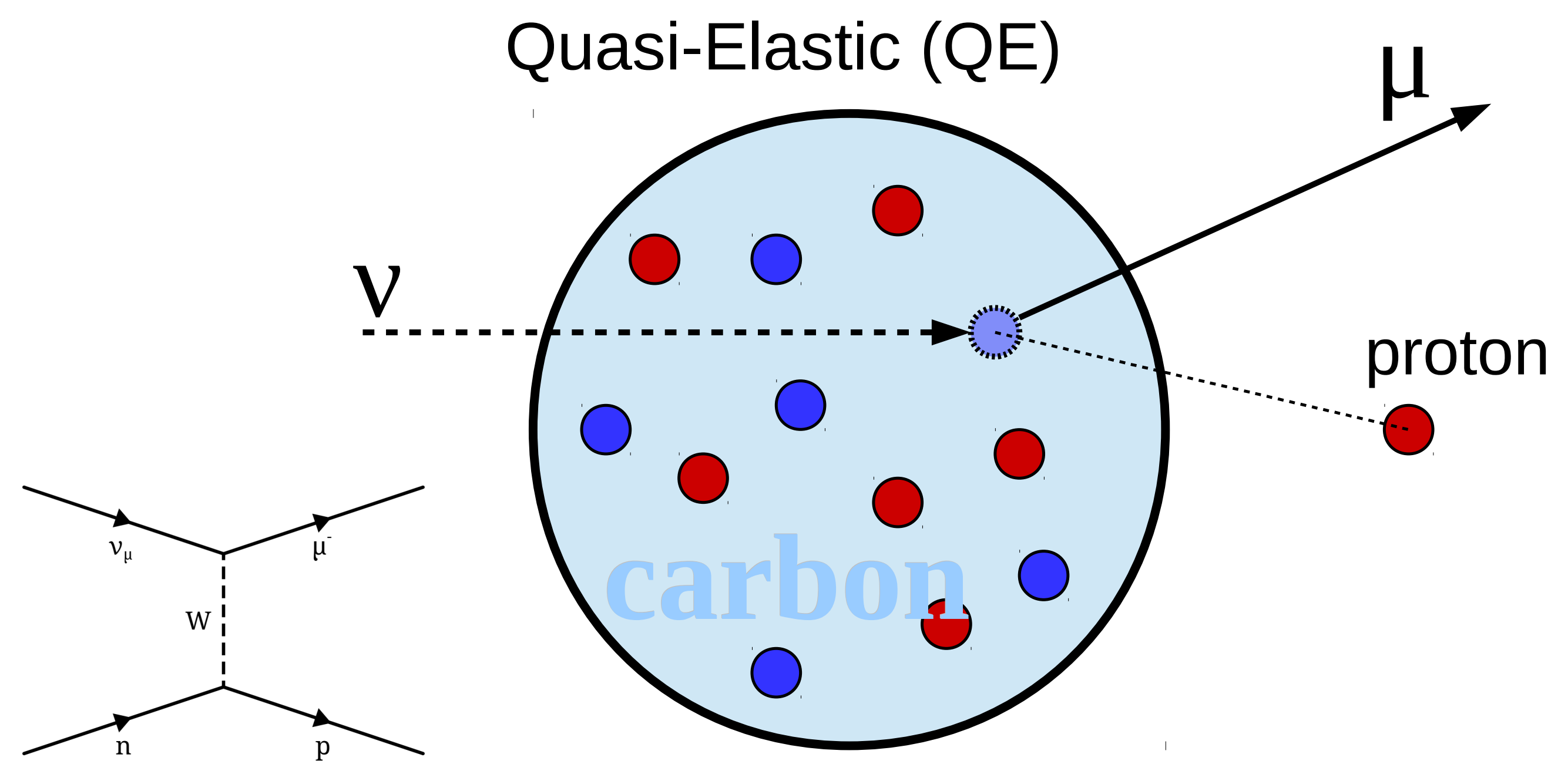


2 Particle, 2 Hole $(2 \mathrm{P} 2 \mathrm{H})$

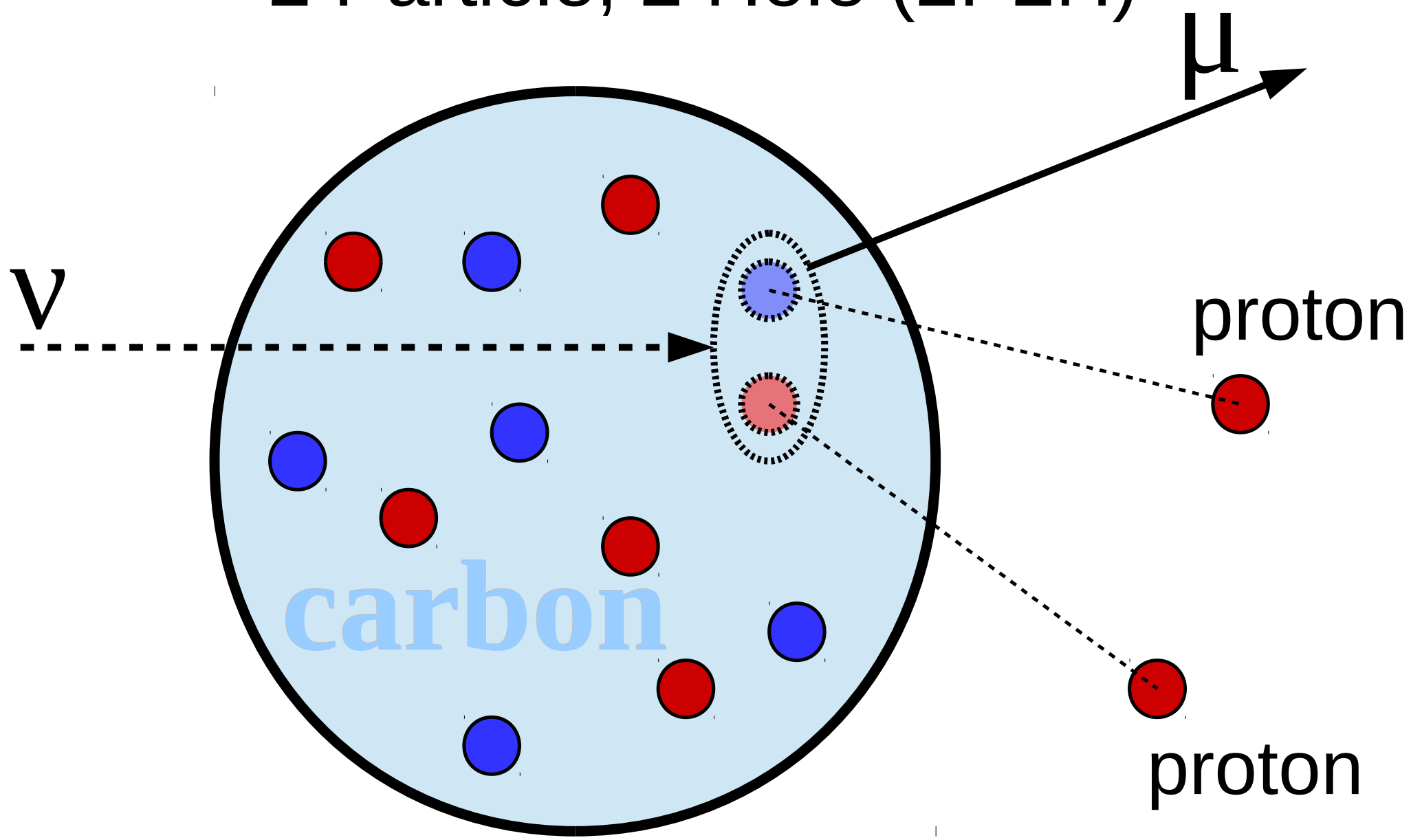




\section{Final State Interaction}

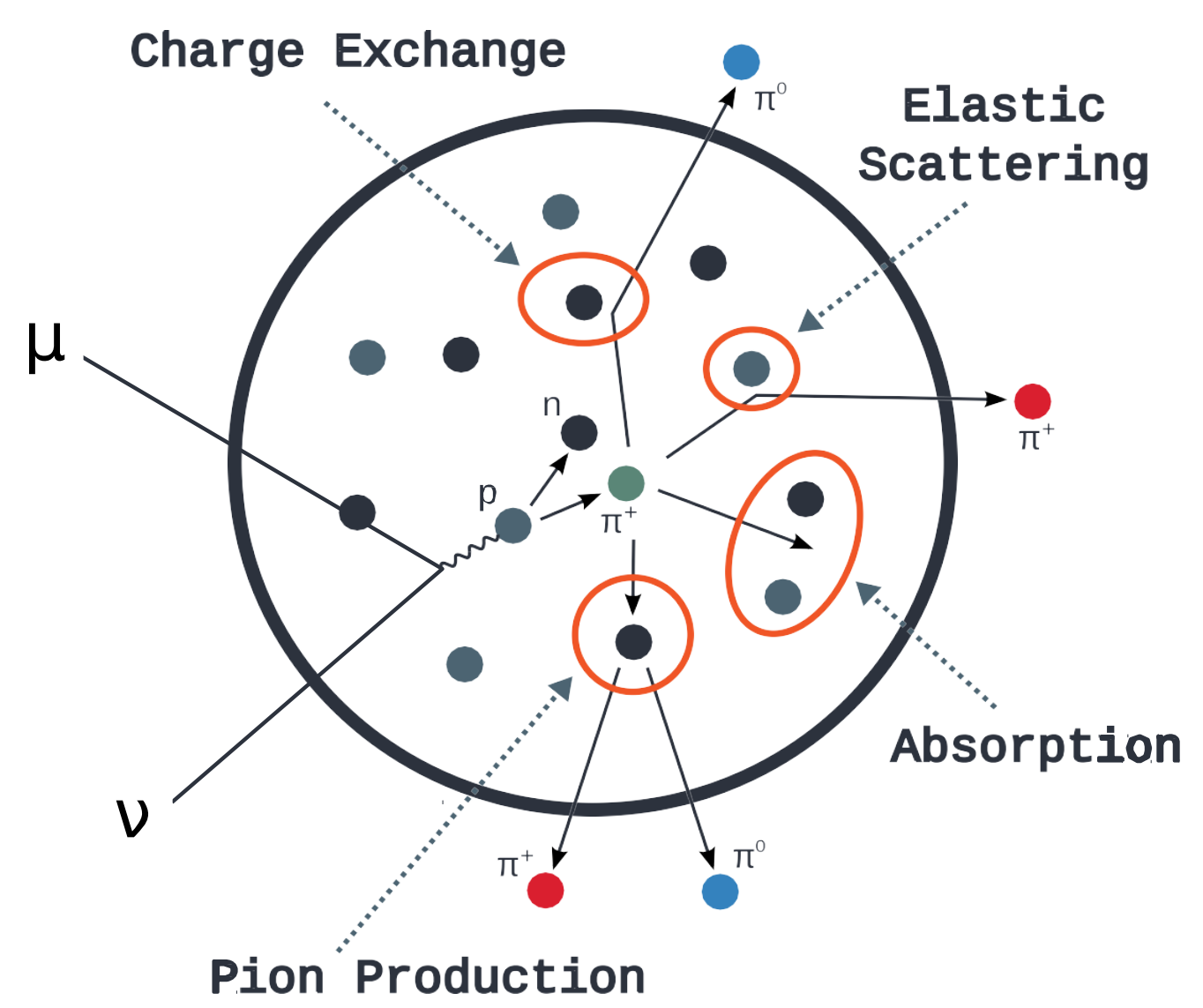

- Initial hadron shower interact within the nucleus changing

- apparent final state configuration

- detected energy.

- An initial pion can charge exchange or be absorbed on a pair of nucleons. The final state observed is $\mu+p$ that makes this a fine candidate for QE production

- We've probably also lost measurable energy 